\title{
¿cómo medir polvos superfinos?
}

\author{
how to measure super-fine pouders?
}

(aRock Produets», agosto 1959, pag. 131.)

W. WIELAND

Según el autor, parece haber encontrado una fórmula que satisface las exigencias de la porosidad y la propiedad de áreas superficiales para el cemento, en el intervalo de 200 a $12.000 \mathrm{~cm}^{2} / \mathrm{g}$. Puede establecerse como sigue:

$$
\mathrm{S}_{\mathrm{v}}=\mathrm{C}\left[t_{*} \cdot e^{\alpha^{\prime}\left(\mathrm{E}_{o}-\mathrm{E}\right)}\right]^{1 / s}
$$

donde

$\mathbf{S}_{v}=$ relación de superficie a volumen de una particula.

C = Constante.

$t_{r}=$ tiempo reducido para lechos de polvo de altura constante, $h_{a}$.

$e=$ base de los logaritmos naturales.

$a^{*}=2,303 a$, siendo $a$ una constante - del material.

$\mathrm{E}_{o}=$ constante de porosidad.

$\mathrm{E}=$ una porosidad específica.

Para el fabricante de cemento es de excepcional importancia conocer las propiedades superficiales del material. Como regla general, los problemas de molienda e hidratación del cemento se resuelven aplicando el aparato de Blaine $u$ otros similares. En la práctica, este método de medida es satisfactorio como ensayo de rutina para el control del proceso de molienda.

Si se trata de polvos extremadamente finos, hay divergencia acerca de la aplicación del método de permeabilidad.

Las investigaciones teóricas y experimentales de las ecuaciones semi-empíricas y la fórmula de R. L. Blaine conducen a los siguientes resultados:

- La superficie específica para un polvo, de acuerdo con $\mathbf{R}$. L. Blaine, depende de la porosidad del lecho de polvo.

- La ley de aditividad para áreas superficiales calculadas para una mezcla de polvos, de acuerdo también con Blaine, no se cumple.

- La fórmula de Blaine satisface las exigencias del control en la molienda de cemento hasta una superficie específica de $3.000 \mathrm{~cm}^{2} / \mathrm{g}$.

- Para valores Blaine superiores a $3.000 \mathrm{~cm}^{2} / \mathrm{g}$, la superficie especifica comienza a depender de la porosidad.

Al comparar la porosidad de un lecho de polvo con su permeabilidad, deriva una 
fórmula, perfectamente empírica, que establece la independencia de la superficie especifica de la porosidad, dentro del intervalo examinado, cumpliendo, a la vez, la ley de aditividad.

Puede establecerse:

$$
\log t_{r}=a \mathbf{E}+b
$$

donde:

$t_{t}=$ tiempo reducido de flujo a través de los lechos de altura constante, $h_{o}$.

$a=$ constante del material.

$b=$ grado de finura que caracteriza al polvo.

$\mathrm{E}=$ porosidad del material.

La fórmula de Blaine no contiene ninguna expresión respecto al grado de compacidad del lecho de polvo. Para evitar la posibilidad de errores serios al usar el aparato de Blaine, el peso del polvo de cemento debe ser siempre de 2,7 gramos. De aquí, para un peso específico medio de $3,16 \mathrm{~cm}^{2} / \mathrm{g}$, la porosidad $\mathrm{E}$ será $=0,5485$.

De acuerdo con las normas A. S. T. M., el peso del polvo no debe ser constante; pero la porosidad será 0,50 y 0,53 para cementos normal y fino, respectivamente. Estos son equivalentes, si permanece constante el peso específico.

En la práctica puede examinarse por este procedimiento cualquier cemento con una superfice especifica de 2.500 a 4.000 $\mathrm{cm}^{2} / \mathrm{g}$. Sin embargo, debe tenerse en cuenta un error adicional debido a la fórmula para superficies específicas mayores de $3.000 \mathrm{~cm}^{2} / \mathrm{g}$, aunque esto tendrá una influencia mínima sobre los resultados de las medidas.

Para polvos más finos y más gruesos, estas prescripciones quedan limitadas por las siguientes circunstancias: los polvos finos no pueden compactarse a una porosidad de 0,55 (ver fig. 3); para polvo grueso, la compacidad del lecho es pequeña para una permeabilidad de $0,5 \mathrm{y}$ la fórmula [2] no es ni remotamente válida (esta-dificultad se evita haciendo el polvo lo más compacto posible).
El principio de la ecuación de permeabilidad, perfectamente empírica, viene dado por la fórmula [2]. Hasta la fecha no puede Interpretarse la relación lineal entre $\log t$, por un lado y $\mathrm{E}$ por otro. En tanto se encuentre una explicación clara, es materia a discutir si se trata de una ley lineal o muy aproximadamente lineal.

Hay límites en la validez de la ley empirica [2]. Al considerar la posible aplicación de la ecuación, debe tenerse en cuenta que:

- Un lecho de polvo no puede compactarse al azar sin destruir las partículas.

- Un relleno no puede elaborarse holgadamente al azar, ya que las partículas siempre tendrán una tendencia a yacer unas sobre otras debido a la gravedad. Aunque no existiese la gravedad, las particulas se moverian por una corriente de aire y abandonarían asi su posición original.

Si examinamos la ecuación [2], obtendremos las siguientes condiciones extremas:

$$
\mathbf{E} \rightarrow 1 ; b \rightarrow \infty ; t_{r} \rightarrow \infty \text {. }
$$

Si $\mathbf{E}=\mathbf{O}$, la célula de permeabilidad se llenará completamente con una sola partícula. Por lo tanto, es superflua la discusión, ya que la ecuación [2] no define $a$ y es sólo válida para polvos.

Después de lograr caracterizar un lecho de polvo por las dos cantidades $a \mathrm{y} b$, se emplearán de acuerdo con $t$ y $\mathbf{E}$ para determinar la superficie especifica.

Gracias a la constante de porosidad $\mathbf{E}_{\jmath}$ es posible una comparación de los diferentes lechos de polvo. De [2] se deduce, como caso general:

$$
t_{r}=e^{a r+b r},
$$

donde

$$
a^{*}=2,303 a \quad \text { y } \quad b^{*}=2,303 b ;
$$

para una porosidad $\mathbf{E}_{n}$

$$
t_{e 0}=e^{a \prime \prime+b s} \text {. }
$$

Siendo $a^{\gamma}$ y $b^{\prime}$ constantes, para el mismo tipo de polvo, podemos escribir:

$$
t_{e o}=t_{r} \cdot e^{a^{\prime}\left(E_{p}-\mathrm{E}\right)}
$$


Si se examinan clínkeres o cemento, sólo permanecerá constante $a^{\prime}$.

La superficie especiffica a determinarse cumplirá con las siguientes condiciones:

- Independencia de la porosidad del lecho de polvo.

- Las mezclas de polvos obedecerán la ecuación:

$$
\mathbf{s}_{v}=\sum \frac{\mathbf{s}_{v 1}}{\mathbf{k}_{j}}
$$

donde $K_{1}=$ relación de volúmenes.

Estas condiciones se cumplen por la relación

$$
\mathbf{s}_{y}=\mathbf{C}\left(t_{z o}\right)^{1 / 2}
$$

Sustituyendo [2]

$$
\mathbf{s}_{v}=\mathrm{C}\left[t_{r} \cdot e^{a^{\prime}\left(\mathrm{E}_{o}-\mathrm{E}\right)}\right]^{1 / 2}
$$

Es difícil prever por consideraciones teóricas la influencia de los factores de forma y granulometría del polvo. No pueden conocerse con detalle las condiciones de flujo del medio a través del lecho de polvo. Se hicieron grandes esfuerzos por establecer una fórmula empírica que satisfaga los requisitos predominantes.

\section{Método de medida por el aparato Blaine} (ffg. 1).

Se introduce una muestra de $3,5 \mathrm{~g}$ en la célula de permeabllidad $D$; se compacta el lecho de polvo poco a poco por medio de un cilindro $\mathbf{S}$, midiendo, para cada altura $h$, el tiempo de flujo $t$ en función de la porosidad del lecho de polvo. Este tiempo se determina como de costumbre, tomando la media de tres determinaciones.

Para la determinación se han de considerar las siguientes correcciones:

\section{Ensayo en blaneo.}

E1 lecho de polvo se halla limitado en sus extremos por dos papeles de filtro. Su importancia en el tiempo de flujo es particularmente notable para polvos de grano grueso, aparentando una permeabilidad más baja. Para igualar desviaciones

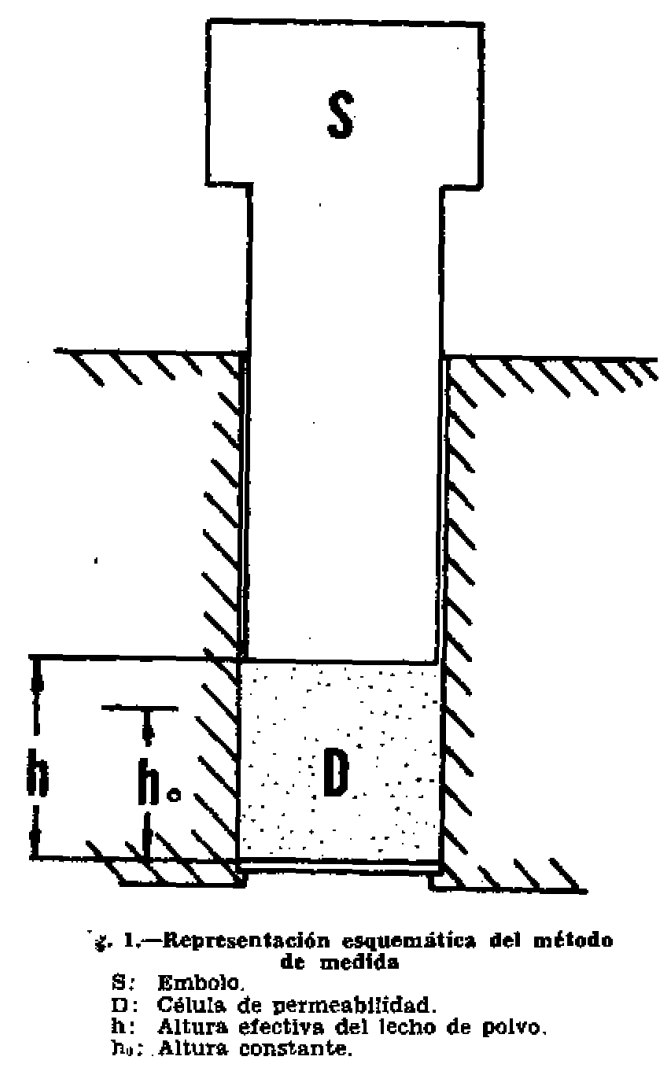

en diámetro y calidad de los discos de papel, se determinó la permeabilidad de 10 de ellos. Para dos discos, el tiempo de flujo $t_{0}=4,6$ segundos.

\section{Reducción a la altura virtual, $h_{0}$.}

Debe de ser posible comparar los tiempos de flujo entre sí; es necesario reducir los tiempos de flujo a lechos de polvo de altura constante $h_{0}$. El tiempo reducido, $t_{r}$, viene dado por la relación:

$$
t_{r}=\frac{h_{n}}{h} \cdot t \text {. }
$$


Si se tiene én cuenta la influencia de los discos de papel de flitro, obtenemos:

$$
t_{r}=\frac{h_{o}}{h}-\left(t-t_{p}\right)
$$

Basados en este método, se examinaron 25 muestras diferentes de clínker y cemento. Para répresentar la función

$$
e=f\left(t_{t}\right)
$$

se empleó un diagrama logarítmico. La figura 2 muestra el tiempo de flujo en función de la porosidad para un cemento standard Holderbank y para un cemento Empa-Standard (de la Swiss Federal Laboratory for Testing Materials and Re- mento investigadas, estas líneas rectas son paralelas, obedeciendo a la ecuación [2].

En la figura 2 se observa que para lechos poco compactados, los puntos de medida no se encuentran en línea recta. Además, los valores obtenidos para ambas series de ensayos, llevados a cabo con el mismo polvo, están de acuerdo entre sí. Esta divergencia de los valores medidos destaca el hecho de que para un polvo insuffientemente compactado, el compactado no es homogóneo donde hay aglomeraciones de partículas y mayores poros en ciertas zonas del lecho. Por lo tanto, se aparenta una mayor porosidad, dando valores de medida más altos que los que

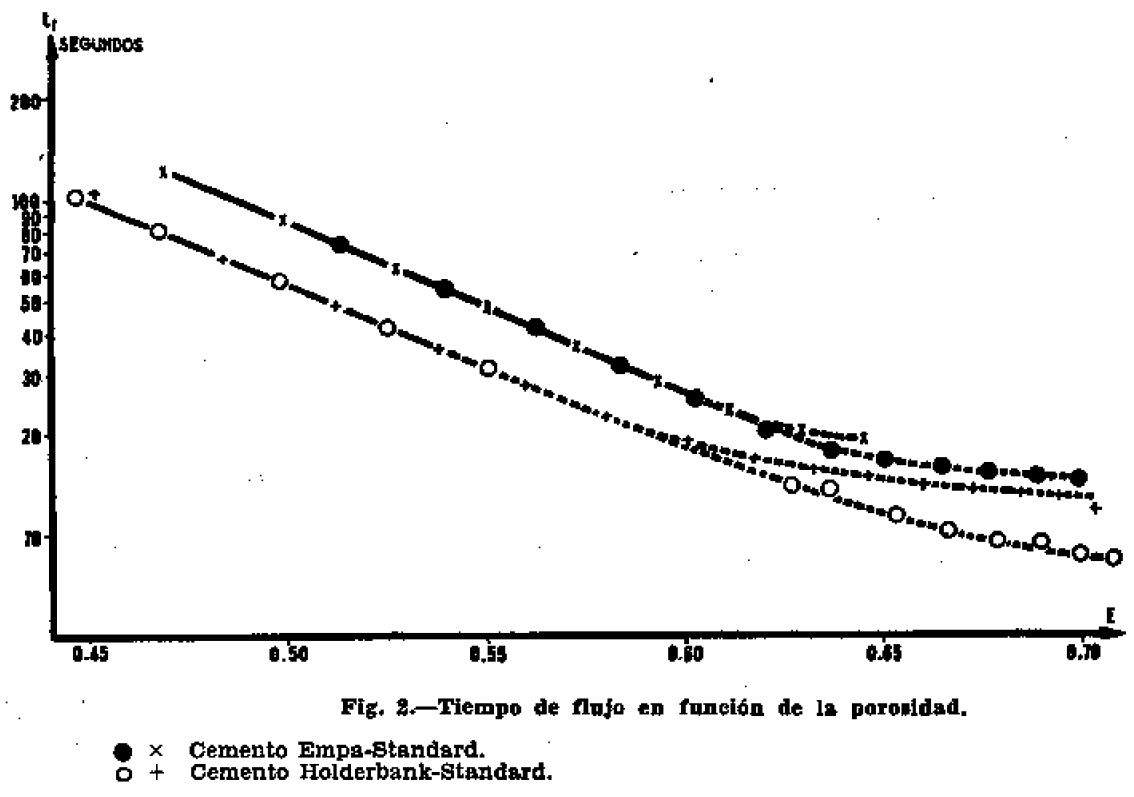

search). Las medidas para cada curva se hicieron dos veces. La figura 3 es un resumen de todas las características.

De las figuras 2 y 3 se deduce:

- A partir de cierta porosidad, según las propiedades del material, la función $e-f\left(t_{*}\right)$ en el diagrama logarítmico es una línea recta.

- Para las muestras de clínker y ce- corresponden a la línea recta del polvo en cuestión.

De la ecuación [1] deriva:

$$
\left.\mathbf{S}_{g}=\frac{\mathrm{C}}{\mathrm{P}} \mid \frac{h_{o}}{h}\left(t-t_{o}\right) \cdot e^{a^{\prime}\left(\mathbb{E}_{o}-\mathrm{E}\right)}\right]^{1 /,}
$$

donde $\mathrm{C}$ no puede determinarse experimentalmente porque no se conoce el área efectiva de la partícula de cemento. Para 
poder determinar $\mathrm{C}$ se ensayaron dos posibilidades:

1. Calibrado con cemento Empa-Standard. De acuerdo con la fórmula [4], en un diagrama $\mathrm{S}_{v}-t_{e o}, \mathrm{C}$ es igual a la pen-

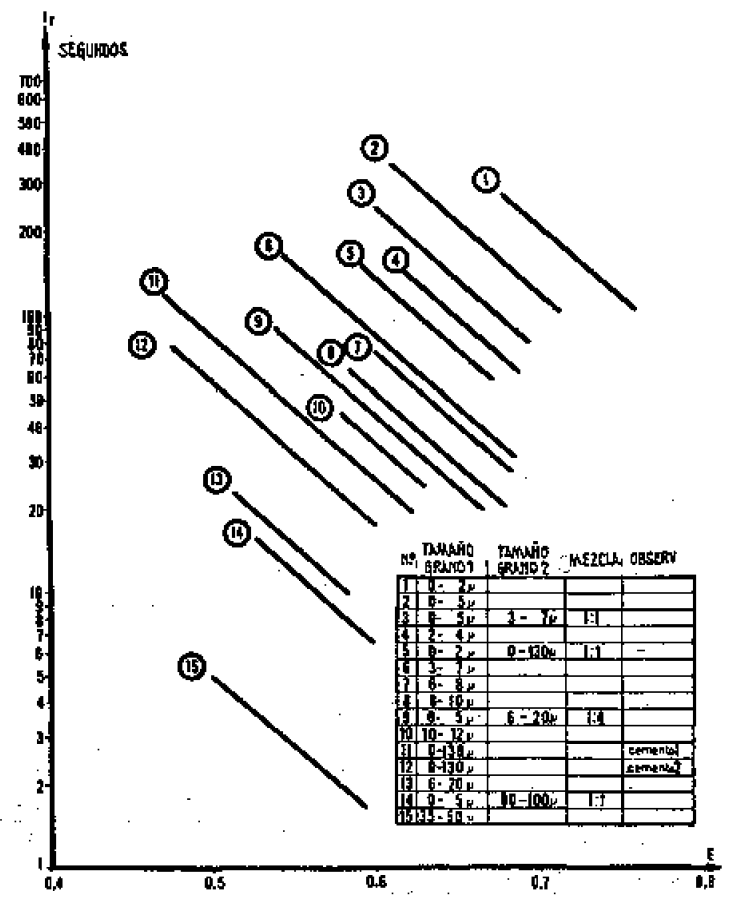

Fig. 3.-Tiempo de flujo en función de la porosidad para Giversas fraccioties $y$ mezelas.

diente de la recta que pasa por el origen. Por lo tanto, es necesario fijar un punto singular en esta recta para determinar $\mathbf{C}$.

EI cemento Empa-Standard tiene un valor Blaine de $3.140 \mathrm{~cm}^{2} / \mathrm{g}$. Como $\rho=$ $=3,16 \mathrm{~g} / \mathrm{cm}^{3}$, Ia superficie especifica por unidad de volumen es igual a: $\mathrm{S}_{w}=\mathbf{9 . 2 2 2}$ centímetros cuadrados / centímetro cúbico.

Además, para $e=0,6:\left(t_{e o}\right)^{1 / 4}=5,13$ $\operatorname{seg} 1 / 4$. y se deduce que

$$
\mathrm{C}_{\mathrm{Empa}}=\frac{\mathrm{S}_{v}}{\left(t_{* o}\right)^{\pi / x}}=1.934,19 \mathrm{seg}-t^{\mathrm{cm}} \mathrm{cm}^{-1}
$$

2. Calibrado con esporas (Lycoperdum piriforme). Estas esporas perfectamente maduras tienen forma globular y un diá- metro de $4 \mu$. Lo mismo que para el clínker, se examinó con estas esporas la relación entre el tiempo de flujo y la porosidad. Como se observará en la figura 4, la recta que determinan las esporas en el diagrama $\log t_{r}-\mathbf{E}$ no es paralela a las rectas para clínker.

$a$ para clínker y cemento $=-5,102$; $a$ para esporas $-4-4,418$; además, para las esporas: $\mathbf{S}_{v}=15.000 \mathrm{~cm}^{2} / \mathrm{cm}^{3}$; para $e=0,6,\left(t_{e o}\right)^{1 / 3}=10,12 \mathrm{seg}^{1 / 2} ;$ de aquí, $\mathrm{C}$ esporas $=1.482,21 \mathrm{seg}^{-1 / 2} \mathrm{~cm}$

Al comparar ambos métodos de calibrado, el factor $\mathrm{C}$ empleando cemento EmpaStandard ofrece la ventaja de que los datos prescritos en las normas para productos comerciales no están sometidos a cambios. A su vez, el emplear un cemento como sustancia de calibrado ofrece la desventaja de las circunstancias que influ-

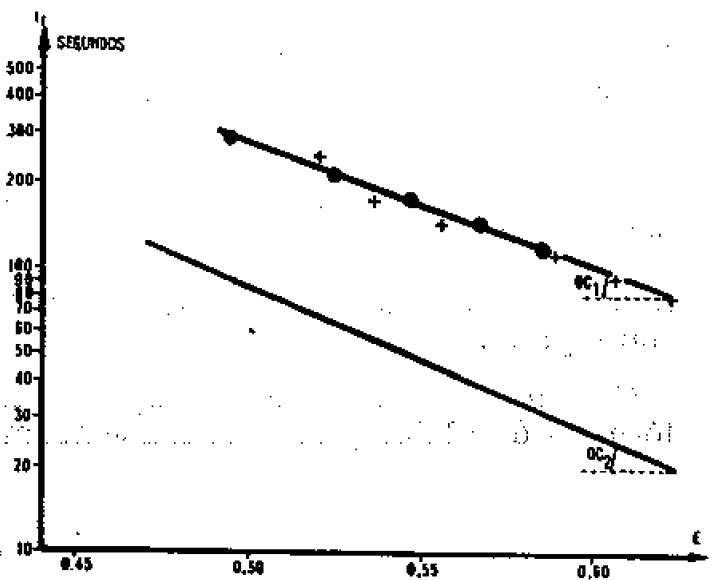

Ffg. 4.-Tiempa de flnjo en funoldn de la porosidad.

Esporas: $a_{1}=\operatorname{tg} a_{1}=-4,418$

Cemento, clinker: $a_{\mathrm{k}}=\operatorname{tg} \mathrm{a}_{\mathrm{A}}=-5,102$

yen las propiedades del material. Además, producir un nuevo cemento standard con la misma superficie específica y la misma distribución granulométrica puede ser difícil.

Es de esperar que las esporas conserven con los años su forma y tamaño sin cambios sensibles. Por este lado, no es necesario un largo almacenamiento. También es interesante referir la: superficie específica 
a una esfera en lugar de a partículas de forma más bien inđefinida. Correspondiendo a la relación de los factores de calibrado $\mathbf{C}_{\mathrm{Empa}} / \mathbf{C}_{\text {epporas }}$, las superficies específicas $\mathbf{S}_{v}$ calculadas con el cemento Empa-Standard como base serán 1,304 veces mayor que en el calibrado con esporas.

En la tabla 1 se comparan ambos valores para áreas superficiales de fracciones distintas al lado de la superficie específica de los correspondientes glóbulos computados para cada intervalo de tamaño de partículas.

La comparación de las superficies específicas de las fracciones separadas determinadas para glóbulos demostraron buen acuerdo con los valores obtenidos usando el factor $\mathrm{C}_{\text {espores }}$, en tanto que el área superficial computada para el factor $\mathbf{C}_{\mathbf{E}_{\text {mpa }}}$ era casi dos veces la magnitud comparada con la superficie de una esfera. Para cuerpos de forma irregular, la superficie específica debe de ser siempre mayor que para una esfera o un cubo. Sin embargo, debe pensarse que la mayor parte de las partículas de clínker no difleren mucho de las formas esférica o cúbica. Por lo tanto, estas consideraciones están en favor del método de calibrado con esporas.

Comparemos la ecuación [1] con la fórmula de Blaine y la ecuación semiem- pirica de J. Kozeny, P. C. Carman y R. L Blaine:

$$
\mathbf{S}_{v}=\left[\frac{\mathrm{A}}{5 \mathrm{~L}_{\eta}}+\frac{\Delta p}{\mathrm{Q}} \frac{\mathrm{E}^{3}}{\left(1-\mathrm{E}^{2}{ }^{2}\right.}\right]^{1 / *}
$$

y con la ecuación perfectamente empirica

$$
\mathbf{S}_{v}=\mathrm{C}\left[\frac{h_{o}}{h}\left(t-t_{o}\right) \cdot \mathbf{E}^{a^{\prime}\left(\mathrm{E}_{o}-\mathbf{E}\right)}\right]^{1 / 2}
$$

A pesar de las discrepancias, ya discutidas, la fórmula de Blaine se ha aprobado en muchos casos. Parece, por lo tanto, que para ciertos intervalos de partículas esta fórmula cubre un amplio margen de adaptación. Como ya hemos dicho, la ecuación semiempírica no puede satisfacer en el caso de cementos finos porque desprecia el flujo molecular. Aún queda por probar la aplicación de esta ecuación a polvos más gruesos.

Sustituyendo en la ecuación [6] $Q=v / t$ (donde $v$ es igual a la cantidad de aire que fluye en el tiempo $t$ ) y conservando las características del aparato así como $\Delta p$ y $v$ que permanecen constantes, obtenemos la ecuación:

$$
\mathbf{s}_{p}=\mathbf{C}^{\prime}\left[t-\frac{\mathrm{E}^{\mathrm{a}}}{(\mathbf{1}-\mathrm{E})^{2}}\right]^{1 / \omega}
$$

\begin{tabular}{|c|c|c|c|c|c|}
\hline \multirow[b]{2}{*}{ Muestro } & \multirow[b]{2}{*}{$\begin{array}{l}\text { Tamanfo } \\
\text { de grano }\end{array}$} & \multicolumn{2}{|c|}{ Sv } & \multirow[b]{2}{*}{$\begin{array}{l}\text { Diámetro } \\
\text { de enferas } \\
(\mu)\end{array}$} & \multirow{2}{*}{$\begin{array}{c}\mathrm{sv} \\
\text { de las esteras } \\
\left(\times 1.000 \mathrm{~cm}^{-1}\right)\end{array}$} \\
\hline & & Por $\underset{\left(\mathrm{cm}^{-1}\right)}{\text { C Emps }}$ & Por $\underset{(\mathrm{cm}-1)}{\text { C eaporss }}$ & & \\
\hline $\begin{array}{l}9001 \\
9002 \\
9003 \\
9004 \\
9005 \\
9006 \\
9007\end{array}$ & $\begin{array}{r}0-2 \\
2-4 \\
4=8 \\
6=8 \\
8-10 \\
10=12 \\
12=14\end{array}$ & $\begin{array}{r}49,960 \\
24,680 \\
23,384 \\
16,557 \\
13,926 \\
11,605 \\
8,433\end{array}$ & $\begin{array}{r}38,286 \\
18,913 \\
17,920 \\
12,688 \\
10,672 \\
8,893 \\
6,462\end{array}$ & $\begin{array}{r}1=2 \\
2=4 \\
4=6 \\
6=8 \\
8=10 \\
10=12 \\
12=14\end{array}$ & $\begin{array}{l}60-30 \\
30-15 \\
15-10 \\
10-7.5 \\
6.0-5.0 \\
5.0-4.3\end{array}$ \\
\hline $\begin{array}{l}D_{4} F_{*} \\
D_{4} F_{1} \\
D_{4} F_{1} \\
D_{4} F_{1} \\
D_{4} F_{3}\end{array}$ & $\begin{array}{r}0-5 \\
2-10 \\
3-20 \\
35-50 \\
50-70\end{array}$ & $\begin{array}{r}38,916 \\
17,562 \\
5,957 \\
2,437 \\
948\end{array}$ & $\begin{array}{r}29,822 \\
13,458 \\
4,565 \\
1,868 \\
728\end{array}$ & $\begin{array}{r}1-5 \\
2-10 \\
3-20 \\
35-50 \\
50-70\end{array}$ & $\begin{array}{l}60-12 \\
30-6.5 \\
20-3.0 \\
1.7-1.2 \\
1.2-0.8\end{array}$ \\
\hline
\end{tabular}

T A B L A 1

Comparación de ambos valores de áreas superficiales para fracciones separadas 
Las ecuaciones [1] y [7] difieren una de otra, por un lado, en las constantes C y C', y, por otro lado, en los factores $E^{s} /(1-E)^{2}$ y $e^{a^{\prime}\left(E_{o}-E\right)}$. Con calibrado

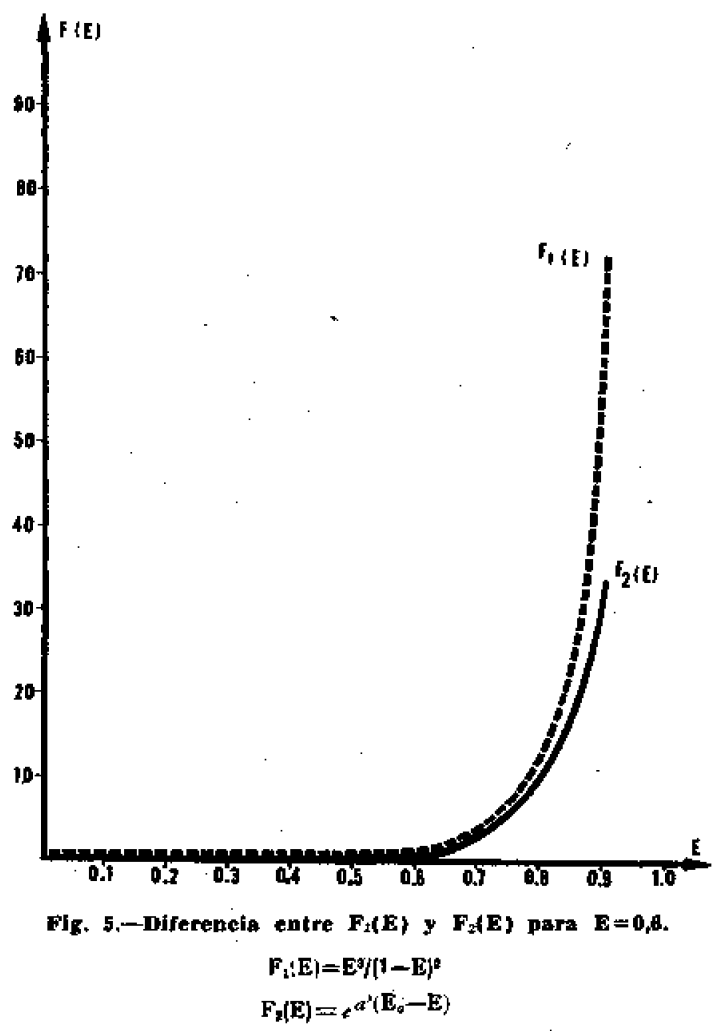

más adecuado de $\mathrm{C}$, es siempre posible hacer $\mathrm{C}^{\prime}=\mathrm{C}$.

La figura 5 representa estas dos funciones $F_{1}(E)$ y $F_{2}(E)$. Estas curvas se parecen mucho entre si, especialmente en el intervalo $\mathrm{E}=0$ y $\mathrm{E}=\mathbf{0}, \mathbf{5}$. La diferencia entre las dos funciones alcanza el valor máximo para el valor límite $E=1$; cuando $F_{1}(E) \rightarrow \infty, E \rightarrow 1 ; F_{2}(E) \rightarrow 109,85$, cuando $\mathrm{E}_{o}=0,6$ y $\mathrm{E}_{1} \rightarrow 1$.

Desistiendo de la relación $\mathbf{C}^{\prime}=\mathbf{C}, \mathbf{E}_{o}$ puede elegirse arbitrariamente. Sin embargo, si $\mathrm{E}_{o}$ asumiese ambos valores límites 0 ó 1, la función $F_{12}(\mathbb{E})$ tendería a alcanzar $\mathrm{E} \rightarrow 1$, un valor finito. Para bajas porosidades y supuesto $\mathrm{C}^{\prime}=\mathrm{C}$, la fórmula [7] daría casi los mismos resultados que la [1]. Las desviaciones se hacen mayores para porosidades crecientes.

De acuerdo con la figura 5 , la diferencia entre $F_{1}(E)$ y $F_{2}(E)$ para porosidades hasta 0,5 , cae dentro del orden de los errores de medida. Como se ve en la figara 3, un cemento con una superficie específica Blaine de $2.500-3.000 \mathrm{~cm}^{2} /$ g puede comprimirse, como máximo, hasta una porosidad $E=0,5$. En consecuencia, la fórmula de Blaine se aplicaría sólo a superficies específicas hasta de $3.000 \mathrm{~cm}^{2} / \mathrm{g}$.

F. S. S.

TA BLA 2

Superftcie específica, de acuerdo con $R$. L. Blaine, en $\mathrm{cm}^{2} / \mathrm{g}$, para tres grados de finura

Númerơ J

$\begin{array}{cccc}.5485 & \mathbf{5 3 1 7} & \mathbf{5 1 5 0} & .4983 \\ & & & \\ \mathbf{2 , 4 4 0} & \mathbf{2 , 4 2 0} & \mathbf{2 , 5 0 5} & \mathbf{2 , 4 4 5} \\ \mathbf{2 , 4 4 0} & \mathbf{2 , 4 2 0} & \mathbf{2 , 4 7 0} & \mathbf{2 , 4 8 5} \\ \mathbf{2 , 4 2 0} & \mathbf{2 , 4 8 0} & \mathbf{2 , 4 7 5} & \mathbf{2 , 4 7 0} \\ \mathbf{2 , 4 5 0} & \mathbf{2 , 5 3 0} & \mathbf{2 , 4 6 5} & \mathbf{2 , 4 5 0} \\ \mathbf{2 , 4 9 0} & \mathbf{2 , 4 8 0} & \mathbf{2 , 5 1 0} & \mathbf{2 , 4 9 0} \\ - & & & \\ 2,448 & \mathbf{2 , 4 6 6} & \mathbf{2 , 4 8 5} & \mathbf{2 , 4 6 8}\end{array}$

Número 2

.5485
$\mathbf{3 , 4 6 0}$
$\mathbf{3 , 4 6 0}$
$\mathbf{3 , 5 3 0}$
$\mathbf{3 , 4 8 5}$
$\mathbf{3 , 4 6 5}$
$\mathbf{3 , 4 8 0}$

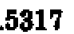

3,520

3,580

3,580

3,580

3,500

3,542
Número 3

$\begin{array}{rrrrr}\mathbf{5 1 5 0} & \mathbf{5 6 5 2} & \mathbf{5 4 8 5} & \mathbf{. 5 3 1 7} & \mathbf{. 5 1 5 0} \\ \mathbf{3 , 6 5 0} & \mathbf{4 , 8 5 0} & \mathbf{4 , 9 8 0} & \mathbf{5 , 0 0 0} & \mathbf{5 , 0 6 0} \\ \mathbf{3 , 5 9 0} & \mathbf{4 , 7 8 0} & \mathbf{4 , 9 9 0} & \mathbf{5 , 0 0 0} & \mathbf{5 , 0 8 0} \\ \mathbf{3 , 6 3 0} & \mathbf{4 , 7 9 0} & \mathbf{4 , 9 5 0} & \mathbf{4 , 9 7 0} & \mathbf{5 , 0 9 0} \\ \mathbf{3 , 5 9 0} & \mathbf{4 , 7 9 0} & \mathbf{4 , 8 7 0} & \mathbf{4 , 9 7 0} & \mathbf{5 , 0 7 0} \\ \mathbf{3 , 6 2 0} & \mathbf{4 , 7 8 0} & \mathbf{4 , 9 4 0} & \mathbf{5 , 0 1 0} & \mathbf{5 , 0 5 0} \\ \mathbf{3 , 6 1 6} & \mathbf{5 , 0 7 0} & \mathbf{4 , 7 9 8} & \mathbf{4 , 9 4 6} & \mathbf{4 , 9 9 0}\end{array}$

